\title{
Acoustic manipulation of electron-hole pairs in GaAs at room temperature
}

\author{
M. M. de Lima, Jr. ${ }^{\text {a) }}$ R. Hey, J. A. H. Stotz, and P. V. Santos \\ Paul-Drude-Institut für Festkörperelektronik, Hausvogteiplatz 5-7, 10117 Berlin, Germany
}

(Received 13 November 2003; accepted 11 February 2004)

\begin{abstract}
We demonstrate the optically detected long-range $(>100 \mu \mathrm{m})$ ambipolar transport of photogenerated electrons and holes at room temperature by surface acoustic waves (SAWs) in (In,Ga)As-based quantum well structures coupled to an optical microcavity. We also show the control of the propagation direction of the carriers by a switch composed of orthogonal SAW beams, which can be used as a basic control gate for information processing based on ambipolar transport.

(C) 2004 American Institute of Physics. [DOI: 10.1063/1.1695636]
\end{abstract}

Recent developments in optical fiber technology have enabled the transmission of information encoded in light pulses over very large distances at rates exceeding several hundreds of GHz. A widespread application of this technology, however, requires efficient techniques to encode, decode, and route the optical signals. Despite considerable advances, purely optic processing techniques are still very limited due to the weak interaction between photon beams, so that information processing is typically performed electronically.

An alternative concept for information processing is based on the conversion of the photons into electron-hole $(e-h)$ pairs in a semiconductor, their manipulation, and subsequent recombination, leading to photon emission. The possibility of interconversion between photons and $e-h$ pairs using surface acoustic waves (SAWs) was demonstrated by Rocke et al. ${ }^{1}$ using low-temperature (i.e., $\sim 10 \mathrm{~K}$ ) photoluminescence (PL) measurements. The electrons and holes generated by photon absorption are captured within the maxima and minima of the moving SAW piezoelectric potential $\left(\Phi_{\mathrm{SAW}}\right)$, respectively. This potential acts as a conveyor belt for the carriers that can then be manipulated during the acoustically induced transport (AIT). Recently, Alsina et $a l .^{2}$ have demonstrated the control of the carrier flow during the AIT at low temperatures using an electronic switch based on interfering SAW beams.

Practical implementation of the previous concepts requires acousto-optic conveyor belts operating at room temperature (RT). Although the RT unipolar AIT of electrons is a well-established process, ${ }^{3}$ the RT ambipolar transport of electrons and holes has been observed only indirectly using electrical techniques ${ }^{4}$ or over very limited distances using optical methods. ${ }^{5}$ The manipulation of the carriers has so far not yet been addressed. In this letter, we present a RT implementation of these concepts using (In,Ga)As-based quantum well $(\mathrm{QW})$ structures. Specifically, we show that $e-h$ pairs produced by photon absorption can be transported with high efficiency over hundreds of microns, after which photons can be retrieved by inducing carrier recombination. In addition, we demonstrate that the flow of electrons and holes at RT can be controlled by interfering SAW beams, which can be

${ }^{a)}$ Electronic mail: mmlimajr@pdi-berlin.de used as basic control gates for solid-state information processing.

The RT-AIT requires an efficient conversion between photons and $e-h$ pairs (and vice versa) as well as low-loss ambipolar transport paths. These two conditions are fulfilled by the structure depicted in Fig. 1, which is composed of a GaAs cavity layer $(\mathrm{C})$ - where the active region, consisting of three $5 \mathrm{~nm}$ thick $\operatorname{In}_{0.2} \mathrm{Ga}_{0.8}$ As QWs spaced by $8 \mathrm{~nm} \mathrm{GaAs}$ barrier QWs is inserted-sandwiched between GaAs/AlAs Bragg mirrors $\left(\mathrm{BM}_{1}\right.$ and $\left.\mathrm{BM}_{2}\right)$. The use of InGaAs/GaAs QW allows the matching of the QW emission and the microcavity resonance at energies well below the GaAs band gap, thus avoiding light absorption by the BMs. The strong confinement of the optical field in the cavity layer, which has an optical thickness corresponding to the QW emission wavelength $\lambda_{L}=945 \mathrm{~nm}$, is essential for an efficient radiative recombination of the $e-h$ pairs after transport. The structure operates at an acoustical wavelength $\lambda_{\mathrm{SAW}}=5600 \mathrm{~nm}$. The Bragg mirrors $\left(\mathrm{BM}_{1}\right.$ and $\left.\mathrm{BM}_{2}\right)$ were grown as described in Ref. 6 in order to maximize the acoustic fields in the cavity region.

The SAWs were generated using focusing interdigital transducers (IDTs) with an angular aperture of $0.1 \mathrm{rad}$. These IDTs produce strong acoustic beams propagating along the [110] direction of the (001) sample surface with a full width at half maximum (FWHM) of approximately three SAW wavelengths and are collimated over distances of several hundred microns. ${ }^{7}$ The $e-h$ pairs are created by a light spot focused at position $G$. Since the potential energy of the transported carriers decreases with the amplitude of the SAW field, the carriers remain inside the beam region during transport. As a result, no further structuring is required to define the transport path.

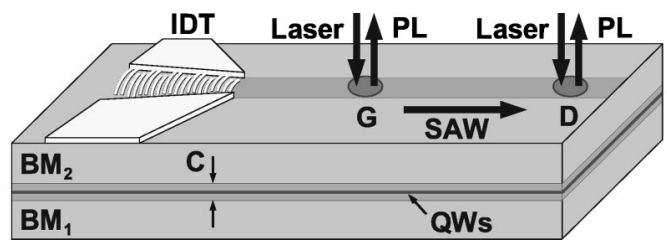

FIG. 1. Sample structure for acoustically induced transport at room temperature. The (In,Ga)As QWs are inserted in an optical cavity $(C)$ sandwiched between Bragg mirrors $\left(\mathrm{BM}_{1}\right.$ and $\left.\mathrm{BM}_{2}\right)$. A focusing IDT generates a strong and collimated acoustic beam, thereby defining the transport path. Light spots at $G$ and $D$ are used to generate and detect the carriers. 


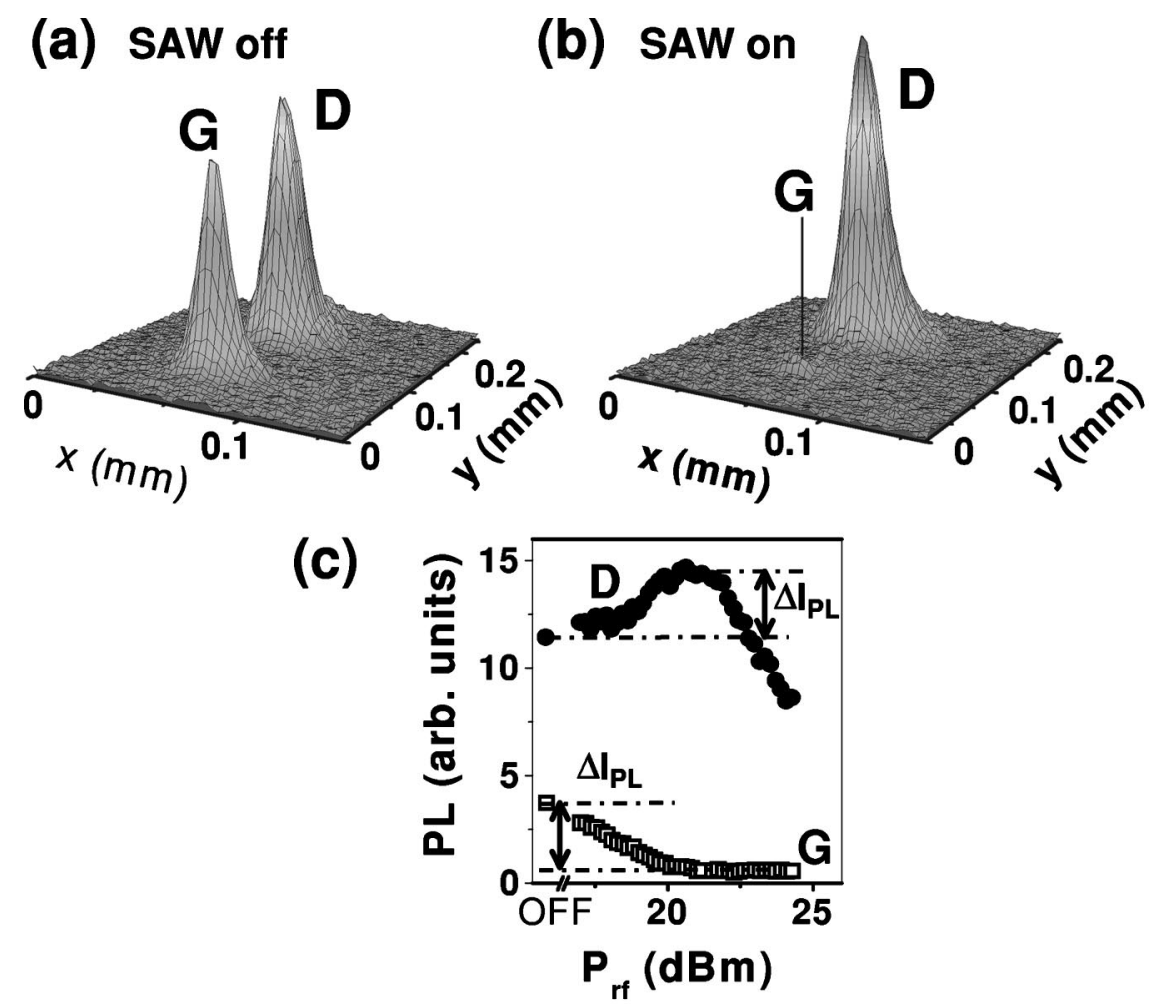

FIG. 2. Spatially resolved PL displayed on a linear scale (a) without a SAW and (b) with a SAW of $P_{\mathrm{rf}}=19.7 \mathrm{dBm}$. (c) Dependence of the integrated intensities at spots $G$ and $D$ (cf. Fig. 1) on the SAW intensity $P_{\mathrm{rf}}$.

The RT-AIT was investigated by exciting the $e-h$ pairs using a focused beam from a tuneable Ti:Sapphire laser operating at $790 \mathrm{~nm}$ and imaging the emitted PL on a chargecoupled-device detector. The emission from the resonant cavity, which is characterized by a single line centered at 945 $\mathrm{nm}$ with a FWHM of approximately $4 \mathrm{~nm}$, was spectrally selected using band pass filters. Although, for practical reasons, the $e-h$ pairs were generated using nonresonant photons, a resonant excitation can be used to increase the conversion efficiency of photons into $e-h$ pairs. The IDTs were excited by continuous radio frequencies (rfs) with nominal power levels $P_{\mathrm{rf}}$ of up to $25 \mathrm{dBm}$. The fraction of the applied electrical power converted into a SAW mode is of only $16 \%$, as determined using a network analyzer.

In order to obtain substantial PL far from the generation spot $G$ to detect the AIT at a remote position $D$, it becomes necessary to quench the piezoelectric field at $D$. This is typically performed by depositing a thin metal stripe on the SAW propagation path to effectively short circuit $\Phi_{\text {SAW }}$ near the surface. Due to the upper Bragg mirror, $\mathrm{BM}_{2}$, the QWs are located in the present case far below the surface, in a region where the screening by a metal stripe is no longer effective. The screening was then provided by the large carrier density generated by a second $790 \mathrm{~nm}$ laser spot focused at $D$. The carriers generated at $G$ and transported by the SAW are then forced to recombine at $D$, thus leading to an increased PL at this spot. The local quenching of $\Phi_{\text {SAW }}$ by the second beam has the disadvantage associated with the inherent PL background from the high density of carriers produced at $D$. It is, however, very flexible, since the detection region becomes defined by a light beam and, thus, provides an additional method to dynamically control the $e-h$ flow. In device applications, the recombination of $e-h$ pairs can also be inDownloaded 02 Apr 2004 to 62.141.165.110. Redistribution subject duced by trapping the transported carriers in potential dots ${ }^{8}$ or wires ${ }^{9}$ positioned in the SAW path.

The RT-AIT is demonstrated in Fig. 2. Figure 2(a) shows the PL profiles in the absence of a SAW and is characterized by peaks at the excitation positions $G$ and $D$ of the two laser spots. The PL intensity at $G$ reduces with increasing SAW amplitude, as shown in Fig. 2(b) and by the squares in Fig. 2(c). The PL quenching at $G$ is attributed to the spatial separation and transport of the carriers by the SAW field away from the generation spot. In contrast, the PL at the detection spot $D$ increases with SAW power up to $22 \mathrm{dBm}$ [cf. Fig. 2(b)]. This enhancement is assigned to the additional recombination of $e-h$ pairs generated at $G$ and transported to $D$ by the SAW field. Finally, for high SAW amplitudes $\left(P_{\mathrm{rf}}>22 \mathrm{dBm}\right)$, the carrier density at $D$ no longer sufficiently screens $\Phi_{\mathrm{SAW}}$, leading to a reduction of the PL intensity at $D$.

In general, the PL intensities at $G$ and $D$ depend on the light fluence as well as on the SAW intensity. The illumination conditions of Fig. 2 (with excitation intensities of the order of $10^{4} \mathrm{~W} / \mathrm{m}^{2}$ ) were chosen so that the PL at $G$ becomes almost completely quenched for $P_{\mathrm{rf}}=20 \mathrm{dBm}$, while the carrier density at $D$ is still sufficiently large to effectively screen $\Phi_{\mathrm{SAW}}$. Under these conditions, the PL enhancement $\Delta I_{\mathrm{PL}}$ at $D$ corresponds closely to the PL intensity at $G$ in the absence of a SAW [cf. Fig. 2(c)]. The carriers are then transported with low losses over the approximately $100 \mu \mathrm{m}$ long path between the two spots.

The previous results demonstrate the interconversion between photons and $e-h$ pairs as well as the efficient longrange ambipolar transport at RT. Another advantage of the AIT is the possibility of controlling the carrier transport direction by combining SAW beams propagating along orto AlP license or copyright, see http://apl.aip.org/apl/copyright.jsp 


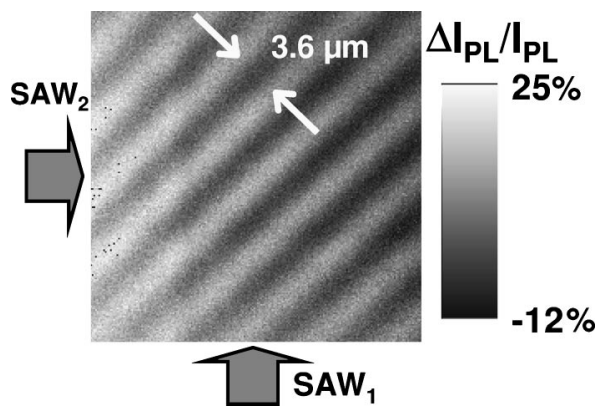

FIG. 3. Normalized difference $\Delta I_{\mathrm{PL}} / I_{\mathrm{PL}}=\left(I_{\mathrm{PL}, \mathrm{SAW}}-I_{\mathrm{PL}}\right) / I_{\mathrm{PL}}$ between the PL intensity under two interfering beams $\mathrm{SAW}_{1}$ and $\mathrm{SAW}_{2}\left(I_{\mathrm{PL}, \mathrm{SAW}}\right)$ and the one in the absence of a SAW $\left(I_{\mathrm{PL}}\right)$. The PL was excited with homogeneous illumination and each SAW beam powered with $P_{\mathrm{rf}}=20 \mathrm{dBm}$. The bright lines oriented at $45^{\circ}$ and separated by $3.6 \mu \mathrm{m}\left(\sim \lambda_{\mathrm{SAW}} / \sqrt{2}\right)$ show the PL emission from moving DDs formed by SAW interference.

thogonal directions. ${ }^{2}$ The interference of the coherent SAW beams leads to the formation of a square lattice of dynamic dots (DDs) with periodicity $\lambda_{\mathrm{SAW}} / \sqrt{2}$, which travel along channels forming an angle of $45^{\circ}$ with the original beams, as recently demonstrated at low temperatures in GaAs QWs. ${ }^{2}$ Carrier transport by DDs is also feasible at RT, as illustrated in Fig. 3, which shows the changes in the PL intensity induced by the intersecting SAW beams. The PL was excited by a wide laser spot that illuminates the whole detection area. The bright lines result from the recombination of carriers trapped and transported by the DDs with extra ones provided by the illumination.

The diagonal propagation of the DDs enables them to transfer carriers between the two interfering beams, as depicted in the upper diagram of Fig. 4(a). A light spot at $G$ is
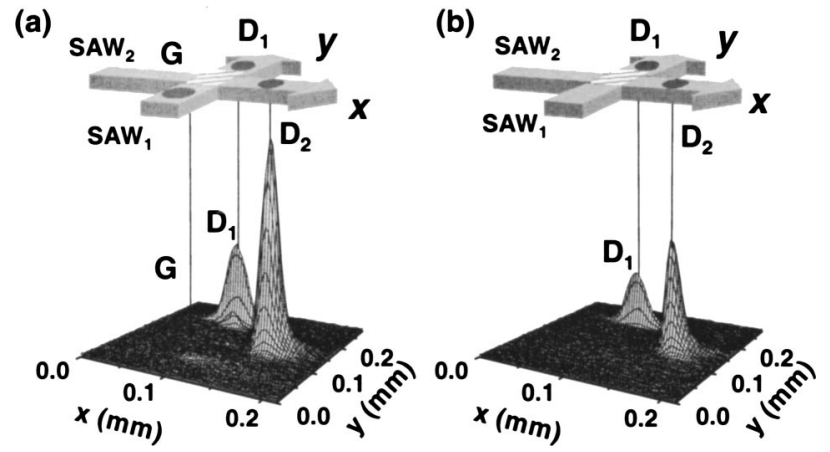

FIG. 4. Carrier switch using orthogonal beams $\mathrm{SAW}_{1}$ and $\mathrm{SAW}_{2}$. When carriers are generated at $G$ (a) the PL intensities (displayed on a linear scale) at positions $D_{1}$ and $D_{2}$ are enhanced as compared with the case (b), where $G$ is not illuminated. Beams $\mathrm{SAW}_{1}$ and $\mathrm{SAW}_{2}$ are excited with $P_{\mathrm{rf}}$ $=23 \mathrm{~dB}$ m each. The upper diagrams illustrate the respective experimental configurations. used to generate $e-h$ pairs in the path of beam $\mathrm{SAW}_{1}$, which transports the carriers to the intersection with the orthogonal beam $\mathrm{SAW}_{2}$. In this region, the DDs transfer a fraction of the carriers to beam $\mathrm{SAW}_{2}$. The AIT is then probed by light spots placed after the intersection at $D_{1}$ and $D_{2}$. In this situation, almost no PL is detected at $G$, since the photogenerated $e-h$ pairs are dissociated and transported away by $\mathrm{SAW}_{1}$. In contrast, the PL intensities at $D_{1}$ and $D_{2}$ become higher than the reference ones in Fig. 4(b), which were detected when the light spot at $G$ was turned off. The enhancement is attributed to carrier transport from $G$ to both $D_{1}$ and $D_{2}$, thus indicating that the DDs in the interference region act as beam splitters for the transported carriers. By using the relative changes in the integrated PL intensities as a measure of the carrier densities, we estimate that approximately $70 \%$ of the carriers photogenerated at $G$ are switched to beam $\mathrm{SAW}_{2}$ and recombine at $D_{2}$, while $30 \%$ remain in $\mathrm{SAW}_{1}$ and recombine at $D_{1}$. This result contrasts with the almost complete switching of the carrier flow to $D_{2}$ observed in low-temperature measurements. ${ }^{2}$ The difference between the two experiments is probably due to the larger carrier diffusion lengths at RT, which tend to spread the charge transported by the DDs in the interference region. ${ }^{10}$

In conclusion, we have demonstrated the RT transport and manipulation of electron and holes by SAWs in (In,Ga)As-based QW structures coupled to an optical microcavity. The principles discussed here may provide the basis for devices for the processing of optical signals based on SAW control of $e-h$ pairs in semiconductors.

The authors thank A. G. Cristóbal and H. T. Grahn for a critical reading of the manuscript and acknowledge the expertise of W. Seidel and S. Krauß in the fabrication of the IDTs.

${ }^{1}$ C. Rocke, S. Zimmermann, A. Wixforth, J. P. Kotthaus, G. Böhm, and G. Weimann, Phys. Rev. Lett. 78, 4099 (1997).

${ }^{2}$ F. Alsina, J. A. H. Stotz, R. Hey, and P. V. Santos, Solid State Commun. 129, 453 (2003)

${ }^{3}$ M. J. Hoskins, H. Morkoç, and B. J. Hunsinger, Appl. Phys. Lett. 41, 332 (1982).

${ }^{4}$ M. Streibl, A. Wixforth, J. P. Kotthaus, A. O. Govorov, C. Kadow, and A. C. Gossard, Appl. Phys. Lett. 75, 4139 (1999).

${ }^{5}$ H. Turk, Ph.D. thesis, Johns Hopkins University, 2002.

${ }^{6}$ M. M. de Lima, Jr., R. Hey, and P. V. Santos, Appl. Phys. Lett. 83, 2997 (2003).

${ }^{7}$ M. M. de Lima, Jr., F. Alsina, W. Seidel, and P. V. Santos, J. Appl. Phys. 94, 7848 (2003).

${ }^{8}$ C. Bödefeld, A. Wixforth, J. Toivonen, M. Sopanen, and H. Lipsanen, Phys. Status Solidi B 224, 703 (2001).

${ }^{9}$ F. Alsina, P. V. Santos, H.-P. Schönherr, W. Seidel, K. H. Ploog, and R. Nötzel, Phys. Rev. B 66, 165330 (2002).

${ }^{10}$ A. García-Cristóbal, A. Cantarero, F. Alsina, and P. V. Santos, Phys. Rev. B (to be published). 